\title{
The Predictive Role of PIK3CA Mutation Status on PI3K Inhibitors in HR+ Breast Cancer Therapy: A Systematic Review and Meta-Analysis
}

\author{
Mingming Wang, ${ }^{1}$ Jin Li, ${ }^{2}$ Jiangsheng Huang, ${ }^{1}$ and Mei Luo $\mathbb{D}^{3}$ \\ ${ }^{1}$ Department of Minimally Invasive Surgery, The Second Xiangya Hospital, Central South University, Changsha, Hunan, China \\ ${ }^{2}$ Department of Anesthesiology, The Third Xiangya Hospital, Central South University, Changsha, Hunan, China \\ ${ }^{3}$ Department of Neurosurgery, Xiangya Hospital, Central South University, Changsha, Hunan, China
}

Correspondence should be addressed to Mei Luo; luomei88@csu.edu.cn

Received 19 February 2020; Accepted 1 May 2020; Published 11 May 2020

Academic Editor: Ji-Fu Wei

Copyright (c) 2020 Mingming Wang et al. This is an open access article distributed under the Creative Commons Attribution License, which permits unrestricted use, distribution, and reproduction in any medium, provided the original work is properly cited.

\begin{abstract}
Aim. To evaluate the impact of PIK3CA mutation status on clinical outcomes of $H R+$ breast cancer treated with $P I 3 K$ inhibitors. Methods. A comprehensive literature search was conducted in online databases from inception to December 31, 2019. The main characteristics and prognostic data of each eligible study were extracted. The odds ratio (OR) for the overall response rate (ORR) and hazard ratio (HR) for progression-free survival (PFS) were estimated using the fixed-effects Mantel-Haenszel model. Results. A total of 8 studies involving 2670 patients were included for analysis. Overall, the clinical outcomes of PI3K inhibitors were significantly influenced by PIK3CA mutation status in $H R+$ breast cancer. After the treatment of PI3K inhibitors, $H R+$ breast cancer patients with PIK3CA mutations presented better ORR (PIK3CA-mutated group: OR = 1.98 [95\% CI, 1.46 to 2.70]; PIK3CA wild-type group: $\mathrm{OR}=1.09$ [95\% CI, 0.78 to 1.53]) and better PFS (PIK3CA-mutated group: $\mathrm{HR}=0.65$ [95\% CI, 0.55 to 0.76]; PIK3CA wild-type group: $\mathrm{HR}=0.87$ [95\% CI, 0.70 to 1.09]). No publication bias was detected for ORR and PFS in our analysis. Conclusion. In this meta-analysis, it suggests that the association between clinical outcomes of PI3K inhibitors and PIK3CA mutation status is dramatic. PIK3CA mutations were a favorable factor in the clinical outcomes of $H R+$ breast cancer treated with $P I 3 K$ inhibitors.
\end{abstract}

\section{Introduction}

Breast cancer is the most commonly diagnosed cancer in women worldwide. More than $80 \%$ of breast cancer is classified as hormone receptor-positive $(H R+)$ breast cancer [1]. Current therapeutic strategies for $\mathrm{HR}+$ breast cancer comprise endocrine therapy, $m T O R$, or cyclin-dependent kinase $4 / 6(C D K 4 / 6)$ inhibition et al. Nevertheless, disease progression, metastasis, and drug resistance eventually develop [2]. To our knowledge, diversified pathways (e.g., RAS/MAPK, $N F \kappa B$, or $P I 3 K / A K T / m T O R)$ get involved in drug resistance to current therapy [3]. Monotherapy targeting a single pathway can be easily failed to generate clinical benefit due to the aberrant activation of bypass signaling. In particular, $P I 3 K / A K T / m T O R$ is the most frequently altered pathway in
$H R+$ breast cancer [4]. Therefore, inhibition of the PI3K/ $A K T / m T O R$ signaling pathway should be of great priority to overcome therapeutic challenges in $H R+$ breast cancer [5].

Previous clinical data have elucidated the clinical efficacy of the PI3K/AKT/mTOR pathway inhibition in $H R+$ breast cancer therapy. For example, administration of everolimus (a rapamycin analog inhibitor of $m T O R$ ) can increase the efficacy of letrozole in the endocrine-resistant form [6]. A combination of everolimus with tamoxifen can improve the survival outcome for $O R+$ metastatic breast cancer patients previously treated with aromatase inhibitors [7]. And the addition of everolimus to exemestane can also improve the survival outcome for breast cancer patients [8]. Besides the $m T O R$ inhibition, oral inhibitors for PI3K isoforms have been established for the treatment of $H R+$ breast cancer. 
Those inhibitors specifically bind the phosphatidylinositol $(3,4,5)$-trisphosphate (PIP3), which mediates the downstream signaling of $P I 3 K$. It has shown synergistic antitumor activity with endocrine therapy against $H R+$ breast cancer in both preclinical and clinical results [9]. Plenty of gene mutations, such as PIK3CA mutations, often occurred in $\mathrm{HR}+$ breast cancer and associated with the clinical efficacy of therapeutic strategies. Based on the results of current research, the $P I 3 K / A K T / m T O R$ pathway can be activated through PIK3CA mutations [10]. Some studies report that PIK3CA mutations can inactivate the downstream components, such as TORC1, which can generate a better outcome in endocrine-resistant breast cancer [11]. Nevertheless, the BOLERO-2 study has debated that there is no association between PIK3CA mutation status and clinical benefit of mTOR inhibition. Similarly, the predictive role of PIK3CA mutation status on the clinical efficacy of $P I 3 K$ inhibitors remains controversial in $\mathrm{HR}+$ breast cancer therapy from current results [12].

According to preclinical and clinical results, whether PIK3CA mutation status can be a predictive role for PI3K inhibitors remains debating. Herein, identification of the predictive role of PIK3CA mutation status on the clinical outcomes of $P I 3 K$ inhibitors should be of great priority to benefit the diagnosis and prognosis for patients with $\mathrm{HR}+$ breast cancer.

\section{Materials and Methods}

2.1. Literature Search Strategy. This meta-analysis was conducted according to the Preferred Reporting Item for Systematic Reviews and Meta-Analyses (PRISMA) statement. We followed the methods of Zou et al. [13]. All reviewed articles were retrieved from PubMed, Cochrane, and Web of Science. Privately and publicly funded clinical studies recorded in ClinicalTrials.gov were also screened. Following terms were applied to comprehensively seize the articles: (1) (Breast Neoplasm) or (Neoplasm, Breast) or (Breast Tumors) or (Breast Tumor) or (Tumor, Breast) or (Tumors, Breast) or (Neoplasms, Breast) or (Breast Cancer) or (Cancer, Breast) or (Mammary Cancer) or (Cancer, Mammary) or (Cancers, Mammary) or (Mammary Cancers) or (Malignant Neoplasm of Breast) or (Breast Malignant Neoplasm) or (Breast Malignant Neoplasms) or (Malignant Tumor of Breast) or (Breast Malignant Tumor) or (Breast Malignant Tumors) or (Cancer of Breast) or (Cancer of the Breast) or (Mammary Carcinoma, Human) or (Carcinoma, Human Mammary) or (Carcinomas, Human Mammary) or (Human Mammary Carcinomas) or (Mammary Carcinomas, Human) or (Human Mammary Carcinoma) or (Mammary Neoplasms, Human) or (Human Mammary Neoplasm) or (Human Mammary Neoplasms) or (Neoplasm, Human Mammary) or (Neoplasms, Human Mammary) or (Mammary Neoplasm, Human) or (Breast Carcinoma) or (Breast Carcinomas) or (Carcinoma, Breast) or (Carcinomas, Breast); and (2) (PIK3CA) or (Phosphoinositide-3-kinase catalytic alpha polypeptide) or (Phosphoinositide-3-kinase catalytic alpha polypeptide). References of included studies were conditionally screened. On-topic articles were included while off-topic items were removed.
2.2. Inclusion Criteria. Eligible studies were (1) patients who were diagnosed with $H R+$ breast cancer, (2) patients who were treated with PI3K inhibitors, (3) the PIK3CA mutation status was detected, and (4) data about the overall response rate (ORR) and progression-free survival (PFS) in PIK3CAmutated group and PIK3CA wild-type group were reported accordingly. Our exclusion criteria were (1) review, letters, comments, conference abstracts, or articles without outcomes of interest and (2) duplicate or overlapping data. If several publications from the same project were identified simultaneously, the newest version and the most comprehensive data would be included.

2.3. Data Extraction and Quality Assessment. Data were independently extracted by two reviewers (Mingming Wang and Jin Li) from the included studies. The following items were extracted from the text and supplementary materials: first author's name, publication year, study type, $P I 3 K$ inhibitor regimen, and specific clinical outcomes. The NewcastleOttawa Scale (NOS) was employed to assess the quality of the included studies. Each study was reckoned according to selection, comparability, and outcome. Any discrepancies were solved by mutual discussion.

2.4. Statistical Analysis. The primary outcomes of interest were ORR and PFS for $H R+$ breast cancer patients in the PIK3CA wild-type group and PIK3CA-mutated group. RevMan 5 (Revman, Cochrane Collaboration; Oxford, England) was applied to perform statistical analysis. The pooled odds ratio of ORR and hazard ratio of PFS were presented to evaluate the difference between the PIK3CA-mutated group and the $P I K 3 C A$ wild-type group. Heterogenicity between studies was assessed by the Chi-squared test and $I^{2}$. A fixed-effects model was applied when there was no significant heterogenicity $\left(I^{2}<50 \%\right.$ or $p$ value $\left.>0.05\right)$. Otherwise, the randomeffects model was in use.

\section{Results}

3.1. Literature Search and Study Selection. The primary search strategy identified 1,642 records after an online inquiry. After removing 945 duplicates and excluding 577 irrelevant records, we included 120 articles after review in the title and abstract. The full text of the remaining 120 articles was meticulously screened and assessed. 112 pieces of literature were excluded due to the following reasons: reviews, case reports, conference abstracts, or without outcomes of interest. Ultimately, 8 studies were selected according to the inclusion and exclusion criteria. Details for the online search strategy were exhibited in Figure 1.

3.2. Main Characteristics of the Included Studies. The main characteristics of the 8 included studies were summarized in Table 1. All included studies were published before December 31, 2019. All patients were diagnosed as $H R+$ breast cancer. The study type of each study and the available register number for each clinical trial were presented. The detailed $P I 3 K$ inhibitor regimen is shown in our table. Six studies were applied to evaluate the survival outcome, and 7 studies were involved to assess the responsive outcome. 


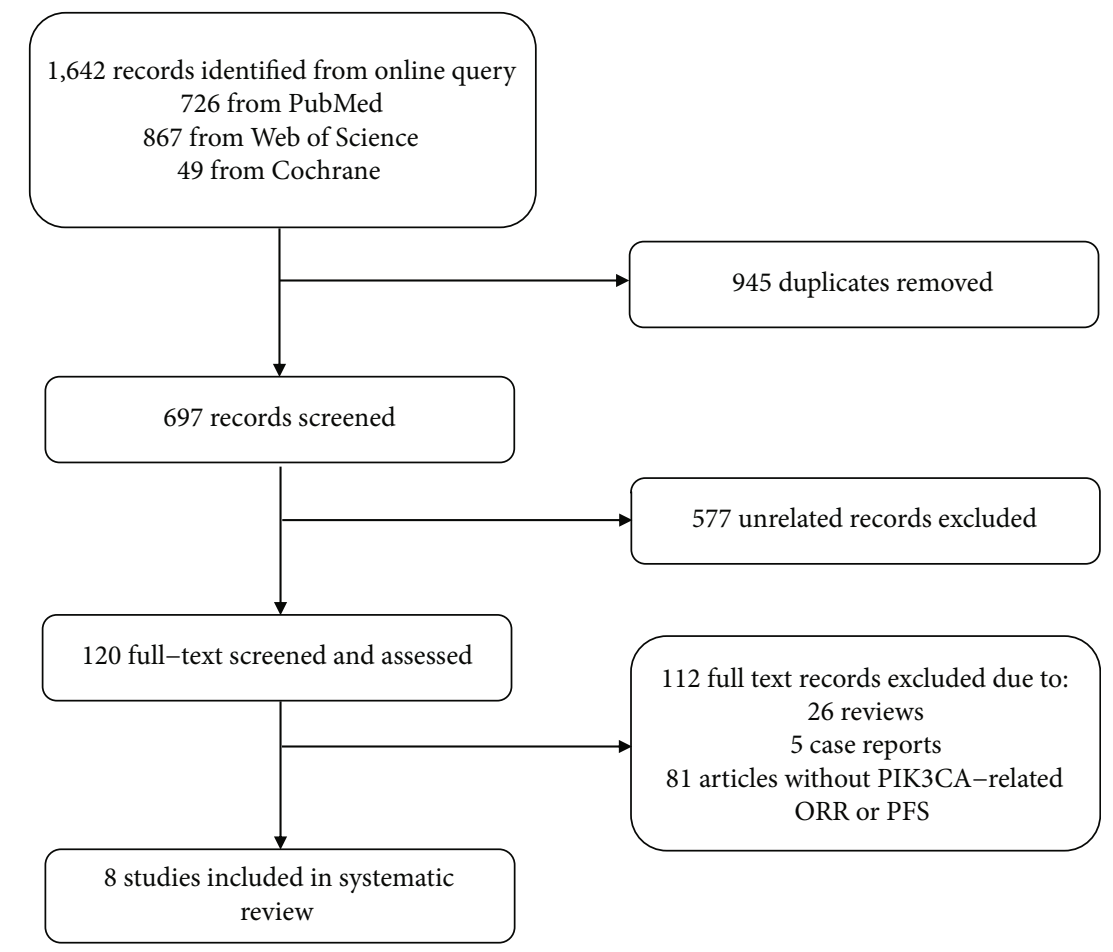

FIgURe 1: Online search strategy.

TABLE 1: Main characteristics of included studies.

\begin{tabular}{|c|c|c|c|c|}
\hline Study and year & Study type & PI3K inhibitor regimen & $\begin{array}{l}\text { Clinical } \\
\text { outcome }\end{array}$ & NOS \\
\hline Krop IE et al. 2016 & Phase 2 trial. NCT01437566 & $\begin{array}{l}\text { Daily pictilisib } 340 \mathrm{mg} \text { plus fulvestrant } \\
500 \mathrm{mg}\end{array}$ & ORR and PFS & 6 \\
\hline Vuylsteke P et al. 2016 & Phase 2 PEGGY study. NCT01740336 & $\begin{array}{c}\text { Daily } 260 \text { mg pictilisib on day } 1-5 \text { each week, } \\
\text { pulsed with paclitaxel }\end{array}$ & ORR and PFS & 6 \\
\hline Loibl S et al. 2017 & $\begin{array}{l}\text { Phase } 2 \text { trial (NeoPHOEBE) } \\
\text { NCT01816594 }\end{array}$ & $\begin{array}{l}\text { Neoadjuvant buparlisib plus trastuzumab } \\
\text { and paclitaxel }\end{array}$ & ORR & 6 \\
\hline Baselga J et al. 2017 & Phase 3 BELLE-2; NCT01610284 & $\begin{array}{c}\text { Daily } 100 \mathrm{mg} \text { buparlisib plus intramuscular } \\
\text { fulvestrant } 500 \mathrm{mg}\end{array}$ & ORR and PFS & 8 \\
\hline Martin M et al. 2017 & Phase 2/3 study. (BELLE-4) NCT01572727 & Daily $100 \mathrm{mg}$ buparlisib with paclitaxel & ORR and PFS & 6 \\
\hline Di Leo A et al. 2018 & Phase 3 BELLE-3 NCT01633060 & $\begin{array}{c}\text { Daily } 100 \mathrm{mg} \text { buparlisib plus intramuscular } \\
\text { fulvestrant } 500 \mathrm{mg}\end{array}$ & PFS & 7 \\
\hline Andre F et al. 2019 & Phase 3 (Solar 1). NCT02437318 & Daily $300 \mathrm{mg}$ alpelisib plus $500 \mathrm{mg}$ fulvestrant & ORR and PFS & 8 \\
\hline Saura C et al. 2019 & Phase 2 trial. NCT02273973 & $\begin{array}{c}4 \mathrm{mg} \text { taselisib ( } 5 \text { days on, } 2 \text { days off) pulsed } \\
\text { with daily } 2.5 \mathrm{mg} \text { letrozole }\end{array}$ & ORR & 6 \\
\hline
\end{tabular}

According to the score, 3 studies had quality scores of 7 or higher while others were less than 7 .

\subsection{Relationship between PIK3CA Mutations and Overall} Response Rate (ORR). A total of 7 studies reported data about the overall response rate (ORR) of $P I 3 K$ inhibitors in the PIK3CA-mutated group and/or PIK3CA wild-type group. Pooled ORR demonstrated that PI3K inhibitors generated 1.98 [95\% CI, 1.46 to 2.70] odds ratio (OR) in $\mathrm{HR}+$ / PIK3CA-mutated breast cancer patients and 1.09 [95\% CI, 0.78 to 1.53 ] odds ratio (OR) in $H R+/ P I K 3 C A$ wild-type breast cancer patients. Medium heterogeneity $(p=0.02$,
$\left.I^{2}=60 \%\right)$ presented in the PIK3CA-mutated group, while no heterogeneity was observed in the PIK3CA wild-type group $\left(p=0.49, I^{2}=0 \%\right)$. The ORR of $P I 3 K$ inhibitors between the PIK3CA-mutated group and the PIK3CA wildtype group was statistically different $\left(p=0.01, I^{2}=84.8 \%\right)$ in Figure 2.

3.4. Relationship between PIK3CA Mutations and Progression-Free Survival (PFS). A total of 6 studies provided data about the progression-free survival (PFS) of PI3K inhibitors in $\mathrm{HR}+$ breast cancer according to PIK3CA mutation status. Comparing with the hazard ratio (HR) of PFS in the 


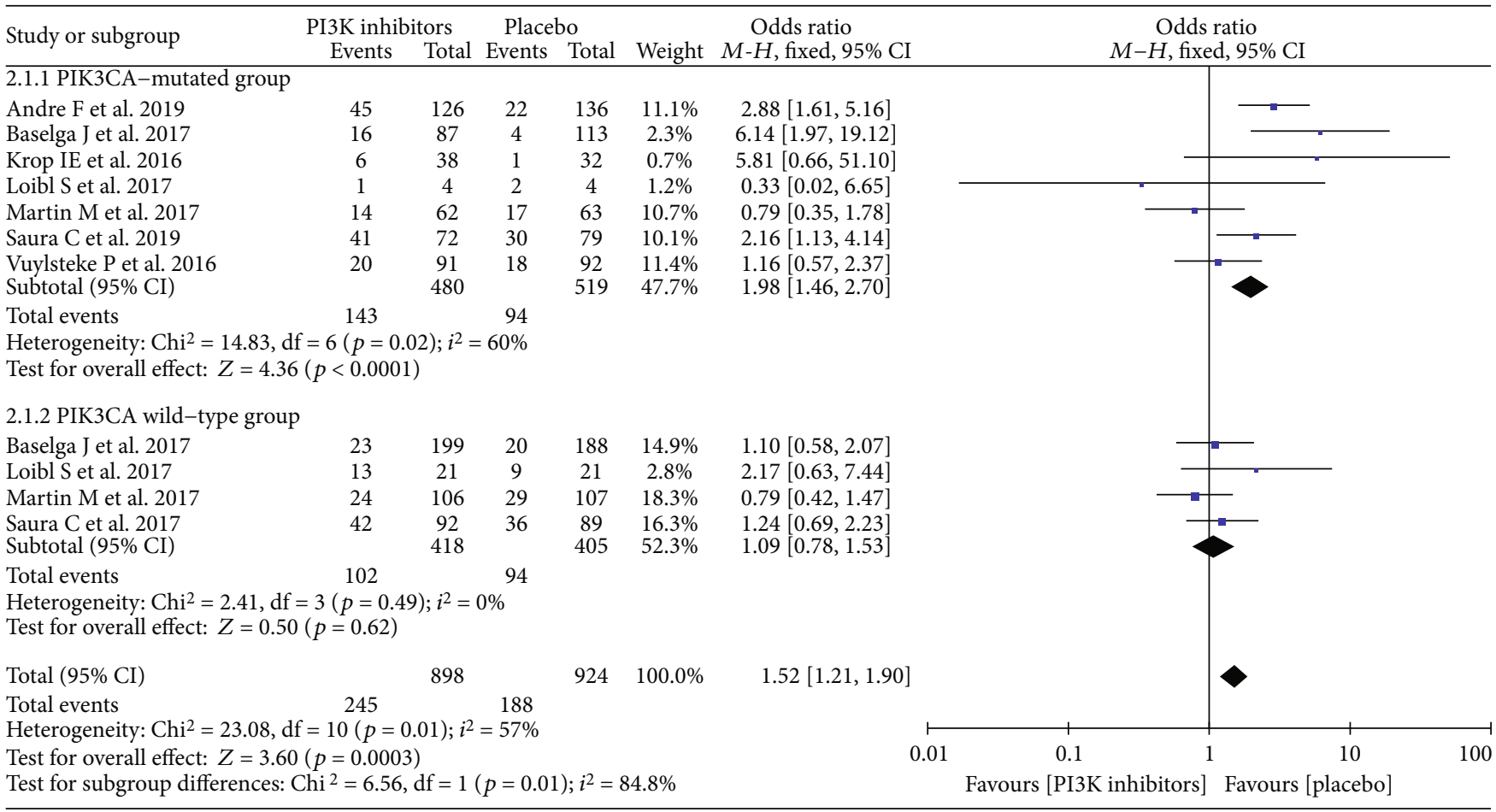

Figure 2: Odds ratio for overall response rate (ORR) in the PIK3CA-mutated group and the PIK3CA wild-type group.

$H R+/ P I K 3 C A$ wild-type group $(\mathrm{HR}=0.87$ [95\% CI, 0.70 to 1.09]), the PFS of $P I 3 K$ inhibitors was further improved in the $H R+/ P I K 3 C A$-mutated group (HR $=0.65$ [95\% CI, 0.55 to 0.76$])$. Medium heterogeneity $\left(p=0.01, I^{2}=66 \%\right)$ existed in the PIK3CA-mutated group, while no heterogeneity was observed in the PIK3CA wild-type group $\left(p=0.64, I^{2}=0 \%\right)$. The improvement of PFS by PI3K inhibitors in the PIK3CAmutated group was significantly better than the PIK3CA wild-type group $\left(p=0.03, I^{2}=77.8 \%\right)$ in Figure 3 .

3.5. Publication Bias. Funnel plots were applied to assess whether publication bias existed in our analysis. The funnel plots did not indicate any publication bias for ORR in Figure 4 and PFS in Figure 5. The visual estimation of the funnel plots showed clear symmetry.

\section{Discussion}

As the most exhaustive meta-analysis to investigate the efficacy of $P I 3 K$ inhibitors in $H R+$ breast cancer according to PIK3CA mutation status, our results demonstrate that the responsive and survival outcomes of $P I 3 K$ inhibitors can be significantly improved in PIK3CA-mutated population comparing with wild-type PIK3CA population. Based on our findings, PIK3CA mutation status can be a prognostic factor for $H R+$ breast cancer patients treated with $P I 3 K$ inhibitors. However, further studies are still required to confirm our findings.

To our knowledge, multiple signaling pathways are implicated in malignant progression and drug resistance in breast cancer. Inhibition of those aberrantly activated pathways is an important therapeutic strategy to manage breast cancer. For example, blockade of the hyperactivated PI3K/AKT/mTOR pathway by diversified inhibitors should bring up some clinical benefits for breast cancer patients. In 2012, mTOR inhibitors (everolimus) showed a profitable effect on endocrine therapy of breast cancer therapy [14]. However, preliminary data on $m T O R$ inhibitors illustrate that $m$ TOR inhibition elicits $A K T$ phosphorylation via feedback activation. AKT phosphorylation often induces drug resistance of $m T O R$ inhibitors. The importance of developing novel functional biomarkers to enhance therapeutic benefit has been highlighted. Treatment with direct PI3K inhibitors could attenuate or abrogate $A K T$ phosphorylation, which might potentially vanquish the resistance to mTOR inhibitors [15].

Latterly, plenty of PI3K inhibitors have been established to treat $H R+$ breast cancer. Current PI3K inhibitors are mainly categorized into 2 types: one is pan-PI3K inhibitors, which targets all four isoforms $(\mathrm{p} 110 \alpha, \beta, \gamma$, and $\delta)$ of $P I 3 K$ (e.g., pictilisib and buparlisib). Another is selective-PI3K inhibitors, which selectively recognize the mutated $P I 3 K \alpha$ subunit and wild-type PI3K (e.g., alpelisib and taselisib) $[16,17]$. The specificity, binding affinity, and efficacy of each inhibitor are varied. According to current reports, pan-PI3K inhibitors often result in dose reduction and discontinuation due to the high rate of severe adverse events $[18,19]$. Rationally, those limitations can be renovated by selective-PI3K inhibitors [20]. A better safety profile permits prolonged administration at higher doses and subsequently advances clinical efficacy [21]. For example, alpelisib is an equipotent selective inhibitor against both the wild-type and mutated $P I 3 K \alpha$ subunit [22]. And taselisib displays greater selectivity for mutated than wild-type $P I 3 K \alpha$ and a less potent 


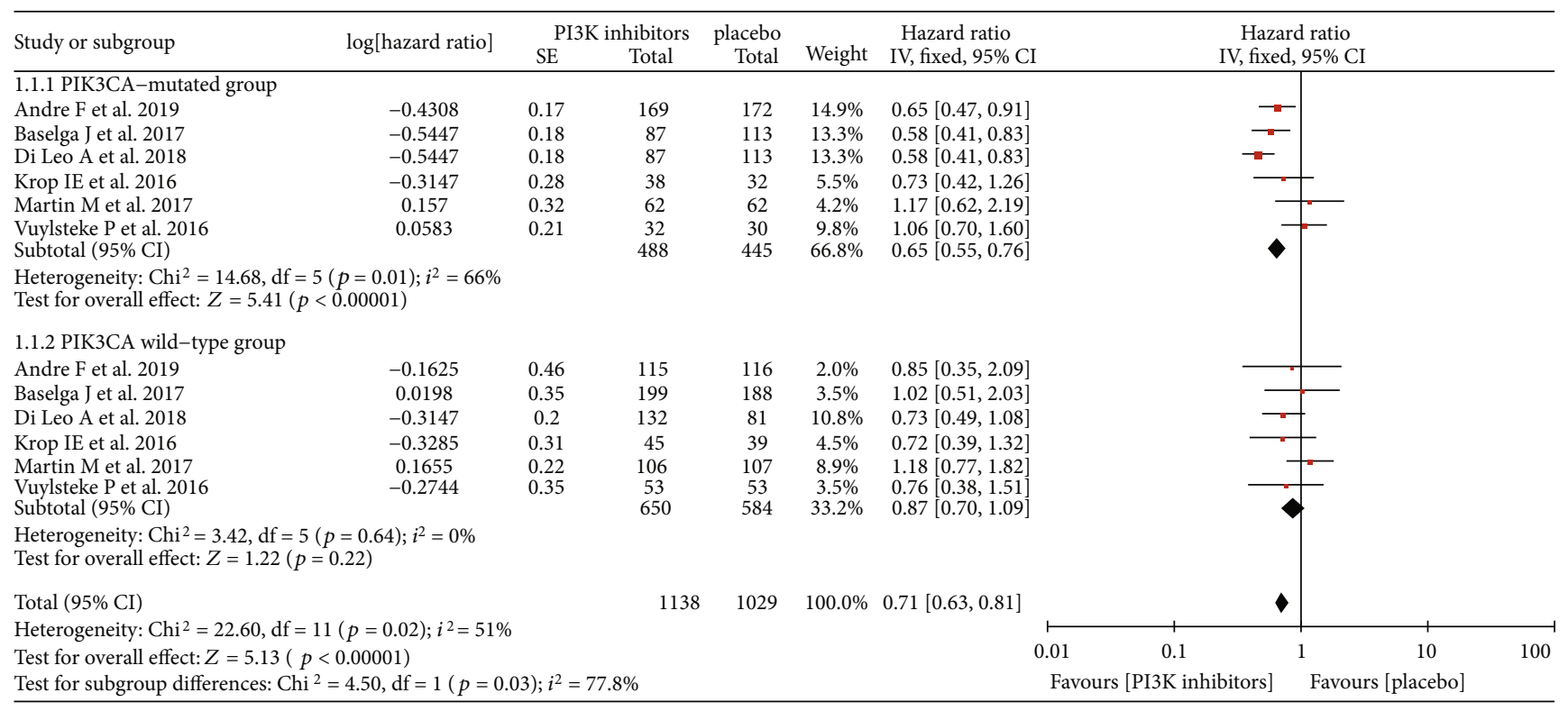

FIgURE 3: Hazard ratio for progression-free survival (PFS) in the PIK3CA-mutated group and the PIK3CA wild-type group.

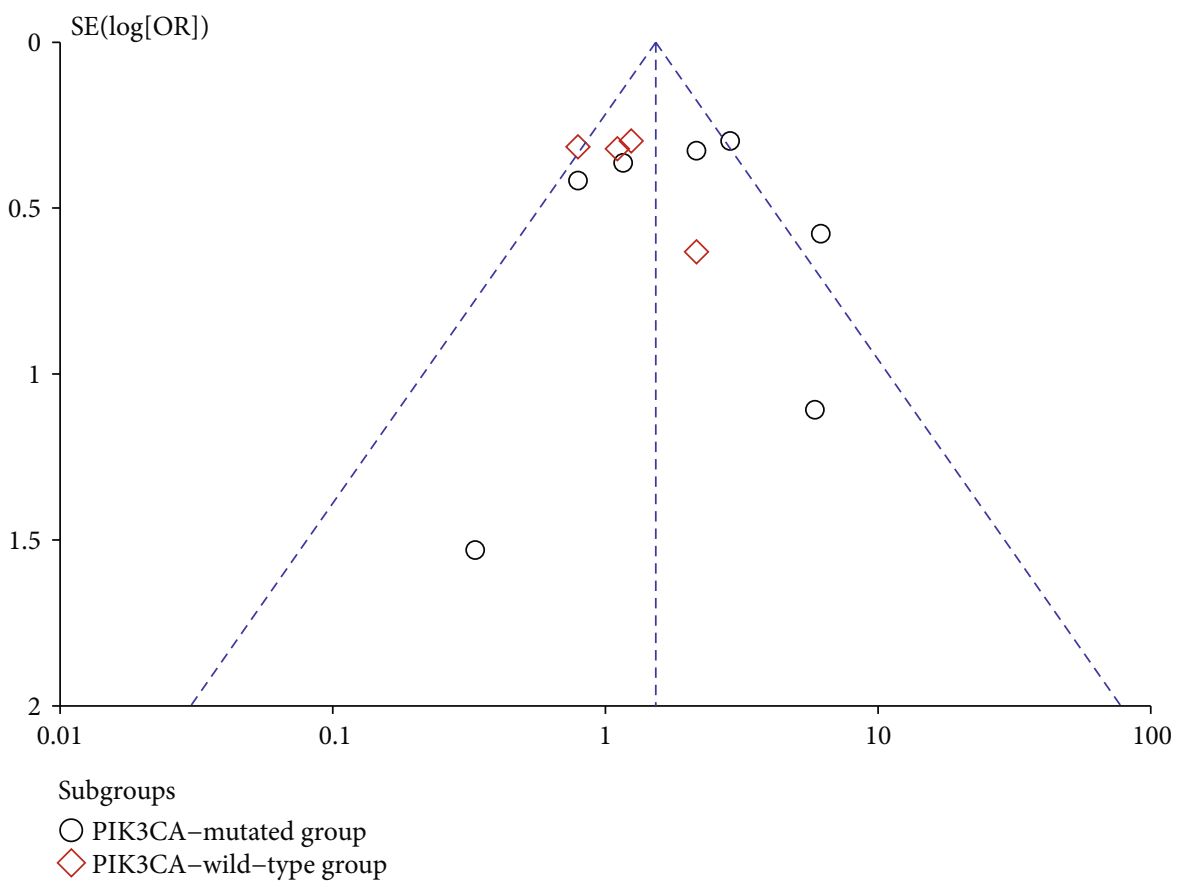

FIGURE 4: Funnel plot for overall response rate (ORR) analysis.

inhibition to $P I 3 K \beta$ isoform [23]. Those selective inhibitors show better clinical benefit not only as a single agent but also in combination with fulvestrant or letrozole. Since the negative clinical results about pan- $P I 3 K$ inhibitors have prevented further investigation, more clinical studies about selective- $P I 3 K$ inhibitors may support our findings on the predictive role of $P I K 3 C A$ mutation status in breast cancer patients [24].

A growing number of clinical studies support that PIK3CA mutation favors the responsive and survival out- come in $P I 3 K$ inhibitor treatment [25], while some studies reported that the clinical efficacy of $P I 3 K$ inhibitors is not affected by the PIK3CA mutation status [26]. The ability of PIK3CA mutation status to predict the benefit of PI3K inhibitors is imperative for further subclassification of $H R+$ breast cancer. A panel of PIK3CA mutations in exons 1, 7, 9, and 20 have been detected and analyzed via current technology (e.g., R88Q in exon 1, N345K in exon 4, C420R in exon 7, E542K in exon 9, E545K/A/G/D in exon 9, Q546K/E/R/L in exon 9, M1043I in exon 20, H1047R/L/Y in exon 20, and 


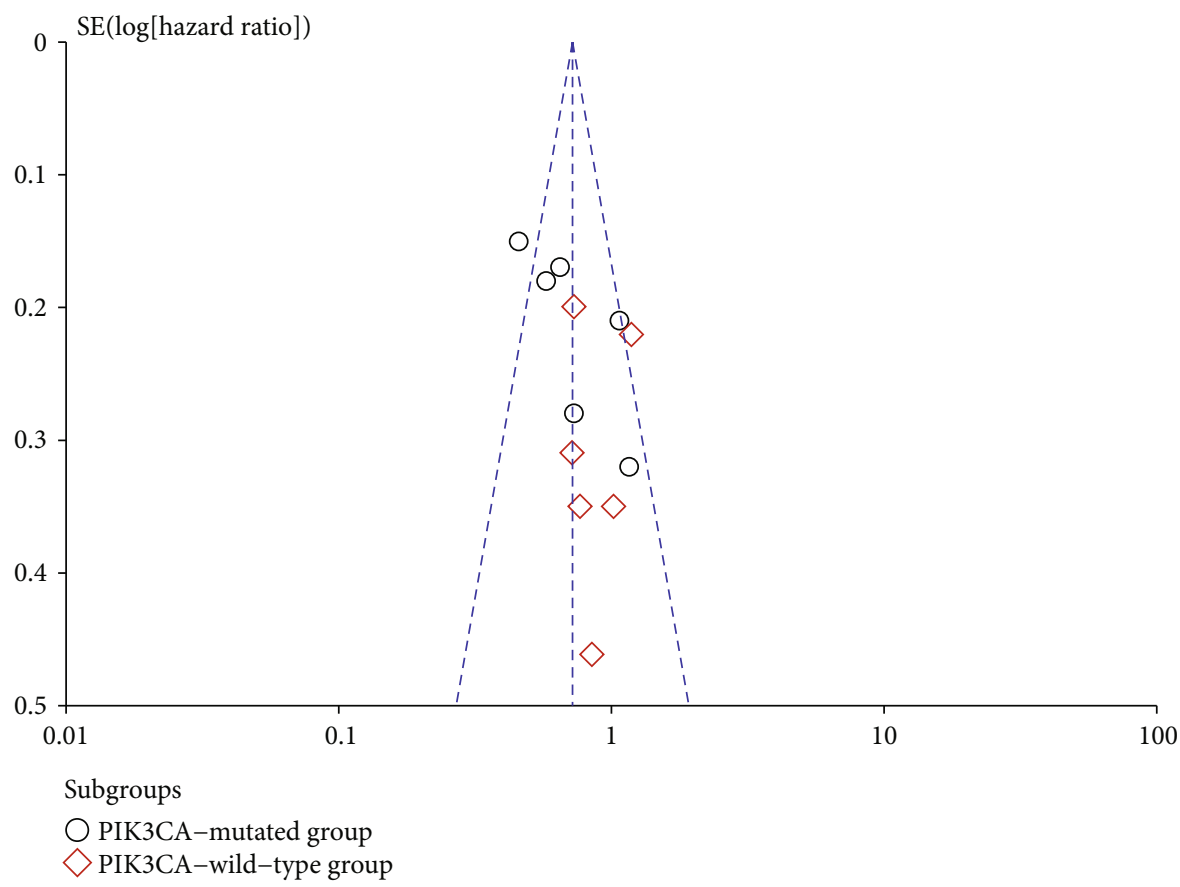

Figure 5: Funnel plot for progression-free survival (PFS) analysis.

H1049R in exon 20) [22]. Notably, the limitation of different strategies to confirm the PIK3CA mutation status should be addressed. Since archived tumors are unlikely to present the real-time PIK3CA mutation status, fresh biopsies are more representative of mutation status [27]. However, additional surgery is not often applicable or desirable in patients with advanced disease or metastasis. Therefore, circulating tumor DNA (ctDNA) analysis has been widely employed as a noninvasive approach to detect PIK3CA mutation status [28]. Comparing with archival tumor samples, ctDNA analysis is a more illustrative and less invasive technique to assess the mutational status and heterogenicity throughout the treatment [29]. In our methods, we collect the responsive and survival data according to the PIK3CA mutation status in ctDNA analysis, which is broadly applied to detect the gene mutation status in cancers.

We have well-demonstrated the favorable role of PIK3CA mutations for $P I 3 K$ inhibitors in $H R+$ breast cancer therapy, whereas our study still has some limitations that should be concerned. Firstly, the disease context, concomitant therapy, and the specificity of each inhibitor should be of great interest in establishing the predictive value of PIK3CA mutation status. Those factors were not indexed by subgroup analysis due to a lack of efficient data. In the future, all of those affective factors should be taken into consideration and well classified. Secondly, the aberrant activation of the PI3K/AKT/mTOR pathway often occurs via various mechanisms, such as the alteration of $P I 3 K$ catalytic subunits (PIK3CA and PIK3CB), $A K T 1, A K T 2$, or PTEN. The crosstalk between PIK3CA mutations with other gene alterations is not discussed in our analysis. Regardless of alterations in the PI3K/AKT/m$T O R$ pathway, a further determination of underlying gene alterations should be of obligation to develop the novel ther- apeutic strategy in breast cancer [30]. The definition of the subsets of breast cancer according to their gene alteration might be essential to maximize the clinical benefit for those patients. Finally, we only assess the responsive and survival outcomes of $P I 3 K$ inhibitors in $H R+$ breast cancer. However, the biological outcomes are surrogate endpoints strongly linked with responsive and survival outcomes [31]. In future studies, the evaluation of those biological outcomes should be an essential supplement to draw a more solid conclusion in this area.

\section{Conclusion}

The PIK3CA mutations are highly associated with better responsive and survival outcomes of $P I 3 K$ inhibitors in $H R+$ breast cancer. The predictive and prognostic role of PIK3CA mutations will facilitate the diagnosis and prognosis of $\mathrm{HR}+$ breast cancer. More studies to explore the clinical superiority of $P I 3 K$ inhibitors are warranted in $H R+/ P I K 3 C A$-mutated breast cancer and other cancer forms.

\section{Data Availability}

The data used and/or analyzed to support the findings in our study are available from the corresponding author upon reasonable request.

\section{Conflicts of Interest}

The authors declare no conflict of interest. 


\section{Authors' Contributions}

Project design was made by MW, JL, and ML. Data collection and analysis were performed MW, JL, JH, and ML. Manuscript preparation and revision were made by MW and ML.

\section{References}

[1] N. Howlader, S. F. Altekruse, C. I. Li et al., "US incidence of breast cancer subtypes defined by joint hormone receptor and HER2 status," Journal of the National Cancer Institute, vol. 106, no. 5, 2014.

[2] G. Rinnerthaler, S. P. Gampenrieder, and R. Greil, "ASCO 2018 highlights: metastatic breast cancer," Memo, vol. 11, no. 4, pp. 276-279, 2018.

[3] J. Shou, S. Massarweh, C. K. Osborne et al., "Mechanisms of tamoxifen resistance: increased estrogen receptor-HER2/neu cross-talk in ER/HER2-positive breast cancer," Journal of the National Cancer Institute, vol. 96, no. 12, pp. 926-935, 2004.

[4] T. W. Miller, B. T. Hennessy, A. M. González-Angulo et al., "Hyperactivation of phosphatidylinositol-3 kinase promotes escape from hormone dependence in estrogen receptorpositive human breast cancer," The Journal of Clinical Investigation, vol. 120, no. 7, pp. 2406-2413, 2010.

[5] N. Vasan, P. Razavi, J. L. Johnson et al., "DoublePIK3CAmutations in cis increase oncogenicity and sensitivity to $\mathrm{PI} 3 \mathrm{~K} \alpha$ inhibitors," Science, vol. 366, no. 6466, pp. 714-723, 2019.

[6] J. Baselga, V. Semiglazov, P. van Dam et al., "Phase II randomized study of neoadjuvant everolimus plus letrozole compared with placebo plus letrozole in patients with estrogen receptorpositive breast cancer," Journal of Clinical Oncology, vol. 27, no. 16, pp. 2630-2637, 2009.

[7] T. Bachelot, C. Bourgier, C. Cropet et al., "Randomized phase II trial of everolimus in combination with tamoxifen in patients with hormone receptor-positive, human epidermal growth factor receptor 2-negative metastatic breast cancer with prior exposure to aromatase inhibitors: a GINECO study," Journal of Clinical Oncology, vol. 30, no. 22, pp. 2718-2724, 2012.

[8] G. Jerusalem, G. Mariani, E. M. Ciruelos et al., "Safety of everolimus plus exemestane in patients with hormone- receptorpositive, HER2-negative locally advanced or metastatic breast cancer progressing on prior non-steroidal aromatase inhibitors: primary results of a phase IIIb, open-label, single-arm, expanded-access multicenter trial (BALLET)," Annals of oncology, vol. 27, no. 9, pp. 1719-1725, 2016.

[9] C. O'Brien, J. J. Wallin, D. Sampath et al., "Predictive biomarkers of sensitivity to the phosphatidylinositol 3' kinase inhibitor GDC-0941 in breast cancer preclinical models," Clinical cancer research, vol. 16, no. 14, pp. 3670-3683, 2010.

[10] K. Kalinsky, L. M. Jacks, A. Heguy et al., "PIK3CA mutation associates with improved outcome in breast cancer," Clinical cancer research, vol. 15, no. 16, pp. 5049-5059, 2009.

[11] S. Loi, B. Haibe-Kains, S. Majjaj et al., "PIK3CA mutations associated with gene signature of low mTORC1 signaling and better outcomes in estrogen receptor-positive breast cancer," Proceedings of the National Academy of Sciences of the United States of America, vol. 107, no. 22, pp. 10208-10213, 2010.

[12] G. N. Hortobagyi, D. Chen, M. Piccart et al., "Correlative Analysis of Genetic Alterations and Everolimus Benefit in Hormone Receptor-Positive, Human Epidermal Growth Factor Receptor 2-Negative Advanced Breast Cancer: Results
From BOLERO-2," Journal of Clinical Oncology, vol. 34, no. 5, pp. 419-426, 2016.

[13] P. Zou, R. Tang, and M. Luo, "Oncolytic virotherapy, alone or in combination with immune checkpoint inhibitors, for advanced melanoma: A systematic review and meta-analysis," International Immunopharmacology, vol. 78, p. 106050, 2020.

[14] J. Baselga, M. Campone, M. Piccart et al., "Everolimus in postmenopausal hormone-receptor-positive advanced breast cancer," The New England Journal of Medicine, vol. 366, no. 6, pp. 520-529, 2012.

[15] J. Baselga, S.-A. Im, H. Iwata et al., "Buparlisib plus fulvestrant versus placebo plus fulvestrant in postmenopausal, hormone receptor-positive, HER2-negative, advanced breast cancer (BELLE-2): a randomised, double-blind, placebo-controlled, phase 3 trial," The Lancet Oncology, vol. 18, no. 7, pp. 904916, 2017.

[16] J. J. Wallin, J. Guan, W. W. Prior et al., "GDC-0941, a novel class I selective PI3K inhibitor, enhances the efficacy of docetaxel in human breast cancer models by increasing cell death in vitro and in vivo," Clinical cancer research, vol. 18, no. 14 , pp. 3901-3911, 2012.

[17] M. Martín, A. Chan, L. Dirix et al., "A randomized adaptive phase II/III study of buparlisib, a pan-class I PI3K inhibitor, combined with paclitaxel for the treatment of HER2advanced breast cancer (BELLE-4)," Annals of oncology, vol. 28, no. 2, pp. 313-320, 2017.

[18] I. A. Mayer, V. G. Abramson, L. Formisano et al., "A Phase Ib Study of Alpelisib (BYL719), a PI3K $\alpha$-Specific Inhibitor, with Letrozole in ER+/HER2-Metastatic Breast Cancer," Clinical cancer research, vol. 23, no. 1, pp. 26-34, 2017.

[19] M. Campone, S. A. Im, H. Iwata et al., "Buparlisib plus fulvestrant versus placebo plus fulvestrant for postmenopausal, hormone receptor-positive, human epidermal growth factor receptor 2-negative, advanced breast cancer: Overall survival results from BELLE-2," European journal of cancer, vol. 103, pp. 147-154, 2018.

[20] C. Saura, D. Hlauschek, M. Oliveira et al., "Neoadjuvant letrozole plus taselisib versus letrozole plus placebo in postmenopausal women with oestrogen receptor-positive, HER2negative, early- stage breast cancer (LORELEI): a multicentre, randomised, double-blind, placebo-controlled, phase 2 trial," The Lancet Oncology, vol. 20, no. 9, pp. 1226-1238, 2019.

[21] S. Loibl, L. de la Pena, V. Nekljudova et al., "Neoadjuvant buparlisib plus trastuzumab and paclitaxel for women with HER2+ primary breast cancer: A randomised, double-blind, placebo-controlled phase II trial (NeoPHOEBE)," European journal of cancer, vol. 85, pp. 133-145, 2017.

[22] D. Juric, F. Janku, J. Rodón et al., “Alpelisib Plus Fulvestrant inPIK3CA-Altered andPIK3CA-Wild-Type Estrogen Receptor-Positive Advanced Breast Cancer," JAMA Oncology, vol. 5, no. 2, article e184475, 2019.

[23] J. Baselga, S. F. Dent, J. Cortés et al., "Phase III study of taselisib $($ GDC-0032) + fulvestrant (FULV) vFULV in patients (pts) with estrogen receptor (ER)-positive,PIK3CA-mutant (MUT), locally advanced or metastatic breast cancer (MBC): Primary analysis from SANDPIPER," Journal of Clinical Oncology, vol. 36, 18_suppl, p. LBA1006, 2018.

[24] F. André, E. Ciruelos, G. Rubovszky et al., "Alpelisib forPIK3CA-Mutated, Hormone Receptor-Positive Advanced Breast Cancer," The New England Journal of Medicine, vol. 380, no. 20, pp. 1929-1940, 2019. 
[25] I. E. Krop, I. A. Mayer, V. Ganju et al., "Pictilisib for oestrogen receptor-positive, aromatase inhibitor-resistant, advanced or metastatic breast cancer (FERGI): a randomised, double-blind, placebo-controlled, phase 2 trial," The Lancet Oncology, vol. 17, no. 6, pp. 811-821, 2016.

[26] P. Vuylsteke, M. Huizing, K. Petrakova et al., "Pictilisib PI3Kinase inhibitor (a phosphatidylinositol 3-kinase [PI3K] inhibitor) plus paclitaxel for the treatment of hormone receptor-positive, HER2-negative, locally recurrent, or metastatic breast cancer: interim analysis of the multicentre, placebocontrolled, phase II randomised PEGGY study," Annals of oncology, vol. 27, no. 11, pp. 2059-2066, 2016.

[27] A. Di Leo, S. Johnston, K. S. Lee et al., "Buparlisib plus fulvestrant in postmenopausal women with hormone-receptor- positive, HER2-negative, advanced breast cancer progressing on or after mTOR inhibition (BELLE-3): a randomised, doubleblind, placebo-controlled, phase 3 trial," The Lancet Oncology, vol. 19, no. 1, pp. 87-100, 2018.

[28] M. J. Higgins, D. Jelovac, E. Barnathan et al., "Detection of tumor PIK3CA status in metastatic breast cancer using peripheral blood," Clinical cancer research, vol. 18, no. 12, pp. 34623469, 2012.

[29] S. K. L. Chia, M. Martin, F. A. Holmes et al., "PIK3CA alterations and benefit with neratinib: analysis from the randomized, double-blind, placebo-controlled, phase III ExteNET trial," Breast cancer research, vol. 21, no. 1, p. 39, 2019.

[30] M. Brandao, R. Caparica, D. Eiger, and E. de Azambuja, "Biomarkers of response and resistance to PI3K inhibitors in estrogen receptor- positive breast cancer patients and combination therapies involving PI3K inhibitors," Annals of oncology, vol. 30, Suppl_10, pp. x27-x42, 2019.

[31] N. Maruyama, Y. Miyoshi, T. Taguchi, Y. Tamaki, M. Monden, and S. Noguchi, "Clinicopathologic analysis of breast cancers withPIK3CAMutations in Japanese women," Clinical cancer research, vol. 13, no. 2, pp. 408-414, 2007. 